\title{
Laser-Generated Localized Freestream Perturbations in Supersonic and Hypersonic Flows
}

\author{
J. D. Schmisseur* \\ U.S. Air Force Research Laboratory, Wright-Patterson Air Force Base, Ohio 45433-7005 \\ and \\ Steven H. Collicott $\mathrm{t}^{\dagger}$ and Steven P. Schneider ${ }^{\dagger}$ \\ Purdue University, West Lafayette, Indiana 47906
}

\begin{abstract}
An optical method for generating localized, controlled perturbations has been developed for use in supersonic and hypersonic flowfields. The thermal spot disturbance is generated when the pulsed beam from a laser is focused at the desired origin and a small region of the gas is ionized. After recombination, the thermal spot persists as a region of heated gas that convects with the local flow velocity. The perturbation is approximately spherical and several millimeters in diameter, with the size dependent on the flow density. Optimal formation of the perturbation is achieved with large-F-number focusing systems. To date, the disturbance has been used as a freestream perturbation for supersonic receptivity experiments as well as forward-facing cavity and blunt-body shock oscillation studies.
\end{abstract}

\section{Introduction}

A $\mathrm{N}$ optical method for generating localized perturbations has been developed for use in supersonic and hypersonic wind tunnels. In addition to the intended use as a freestream disturbance source for boundary-layer receptivity experiments, the laser perturber has been used to investigate the effects of disturbances on other types of flowfields including stagnation regions on blunt bodies and forward-facing cavities. ${ }^{1,2}$ Potential additional applications of the laser perturberinclude velocity measurement and use as an excitationfor the investigationof sensor frequencyresponse. Although developed for use in the Mach-4 Purdue Quiet-Flow Ludwieg Tube, the laser-perturber technique should prove useful in many hypersonic facilities. Because the Ludwieg Tube operates with driver tube stagnation conditions at the ambient conditions in the laboratory, the freestream density is comparable to that encountered in facilities operating at higher Mach numbers.

A schematic of the laser perturber is shown in Fig. 1. A pulsed laser focused at the desired origin of the disturbance is used to photoionize the air locally. The photoionizedregion exists briefly as a plasma and quickly reassociatesto form a small region of heated air referred to as the thermal spot. The ionization process also produces a spherical shock wave that weakens as it propagates away from the central region occupied by the thermal spot. At typical freestream conditions, the shock wave generated by the ionization is very weak, and the thermal spot is the useful portion of the disturbance. Under other conditions, the shock wave generated by the disturbance may also be useful as a perturbation. As indicated in Fig. 1, the thermal spot convects with the local velocity of the flowfield.

The formation of the disturbance depends on the intensity of the laser pulse. Hence, both the energy in the pulse and the size of the focus affect the ionization process. A minimum intensity, known as the threshold intensity, is required to initiate ionization..$^{3-5}$ The air is transparent to the radiation at intensities below the threshold intensity. Once the threshold intensity has been surpassed, increasing intensity results in increasingly strong disturbances.

The two primary ionization mechanisms are multiphoton absorption and cascade ionization. ${ }^{3-5}$ Multiphoton absorption, the dom-

Presented as Paper 98-2495 at the AIAA 20th Advanced Measurement and Ground Testing Technology Conference, Albuquerque, NM, 15-18 June 1998; received 4 February 1999; revision received 10 August 1999; accepted for publication 16 August 1999. This paper is declared a work of the U.S Government and is not subject to copyright protection in the United States.

${ }^{*}$ Research Aerospace Engineer. Senior Member AIAA.

${ }^{\dagger}$ Associate Professor, School of Aeronautics and Astronautics. Senior Member AIAA. inant mechanism at low gas densities, occurs when a molecule absorbs $n$ photons whose combined energy $n h v$ ( $h$ is the Planck constant and $v$ is the light frequency) exceeds the ionization potential of the molecule. Generally, a large number of photons are required. For example, Dewhurst ${ }^{7}$ reports $n=7$ for the ionization of oxygen using 690-nm light. For longer wavelengths, with less energy per photon, the number of photons required for ionization increases. Cascade ionizationis the dominant mechanismat highergas densities. During cascade ionization, electrons gain energy through repeated absorption of photons in the presence of a third body (inverse bremsstrahlung absorption) until the electron has sufficient energy to ionize a molecule directly in a binary collision.

Frequently in the literature, the threshold intensity $I_{\text {th }}$ is related to the gas pressure $P$ as

$$
I_{\mathrm{th}} \propto P^{-\alpha}
$$

where the value of $\alpha$ is determined by the ionization mechanism. ${ }^{8,9}$ Implicit in this relation is the assumption that the gas remains at a fixed temperature. Typically, this has been a valid assumption because the majority of the experimental investigations of laserinduced breakdown seem to have been made in fixed-volume, uninsulated test cells where the gas is at ambient temperature (see, for example, Armstrong et al. ${ }^{8}$ or Dewhurst ${ }^{10}$ ). That the threshold intensity is actually a function of the gas density may be seen in the report by Grey Morgan, ${ }^{5}$ where the threshold intensity is expressed in terms of the neutral molecule concentration for both ionization mechanisms.

Theoretically, when ionization is purely due to multiphoton absorption (for pressures less than $\approx 100$ torr at ambient temperature), $\alpha=1 / n$, where $n$ is the number of photons required to ionize the molecule. ${ }^{5,7}$ Because multiple photons are required, multiphoton ionization typically results in a weak dependence of the ionization threshold on the gas pressure. Cascade ionization, in contrast, exhibits a strong dependence on the gas pressure and the theory predicts that $\alpha \approx 1$ (Ref. 5). In practice, experimental values obtained for $\alpha$ in atmospheric gases range from 0.3 to 0.9 , with the variation in $\alpha$ attributed to the combined effects of both multiphoton and cascade ionization. $8,9,11$

\section{Optical System}

A schematic of the general optical configuration used in this experiment is shown in Fig. 2. A negative lens was used to expand the beam, followed by a positive lens to either collimate the beam at a larger diameter or at least decrease the expansion rate. A final positive lens focused the beam into the test section. The converging beam passed through the window at a small angle with respect to 


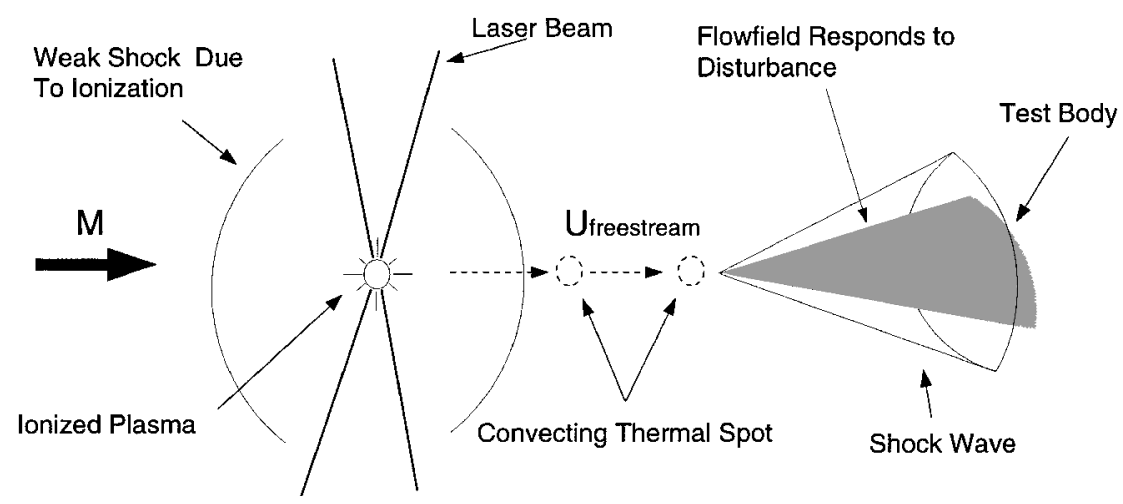

Fig. 1 Major features of the thermal spot perturbation, as applied to receptivity measurements.

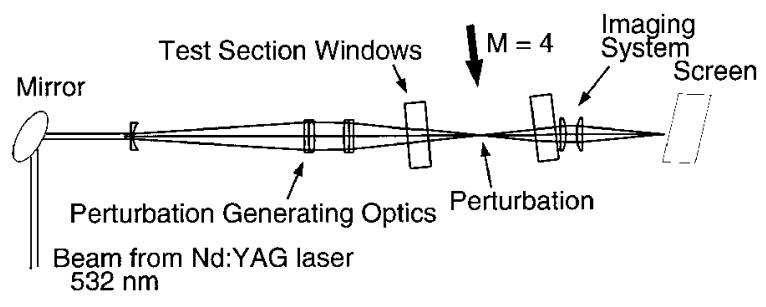

Fig. 2 General optical configuration.

the window normal, approximately $5 \mathrm{deg}$. Although the small tilt increased aberrations, it was necessary to prevent the reflected beam from focusing on the focusing lens and damaging it.

A $10-\mathrm{Hz}$ frequency-doubled (532-nm) Spectra-Physics Model GCR-190 Nd:YAG laser was used to generate the disturbance. The pulse duration was approximately $7 \mathrm{~ns}$, with a maximum pulse energy near $260 \mathrm{~mJ}$. Injection seeding narrowed the emission line width, which temporally smoothed the laser pulse, resulting in increased shot-to-shot consistency. The beam diameter, as quoted by the manufacturer, had a $90 \%$ fit to Gaussian in the near field and $95 \%$ fit to Gaussian in the far field when injection seeded. The $1 / e^{2}$ beam diameter at the laser was measured to be about $4 \mathrm{~mm}$ (Ref. 12).

The laser-perturber optical system was designed using the Zemax ${ }^{13}$ ray-tracing software. The design was optimized for minimum spot root-mean-square(rms) diameter with a constrained total system length. Stock singlet lenses were used for this design, with the lens spacing optimized for minimum beam diameter in the image plane. Note that the combination of the negative and first positive lens did not collimate the beam. Instead, the first two lenses simply expanded the beam before it was focused by the final positive lens.

Focusing performance was quantified via analysis of spot diagrams constructed from the image-plane intercepts of an array of rays originating from a virtual object plane. The array consisted of 135 rays grouped into seven rings whose spacing was set to give a Gaussian distribution about the beam axis, corresponding to the Gaussian intensity profile of the laser. The location of the object plane was determined by the measured beam diameter and the divergence quoted by the manufacturer. Two measures of merit for system performance were used: the Airy radius and the rms spot radius. The Airy radius is the radius of the first dark ring in the diffraction pattern for a uniformly illuminated circular entrance pupil. Thus,

$$
r_{\text {Airy }}=1.22 \lambda F
$$

where $\lambda$ is the laser wavelength and $F$ is the F-number of the converging cone of light. Although with the laser beam the illumination was not uniform, but approximately Gaussian, the Airy radius remains an important characteristic length scale and is still useful as an approximation of the diffraction limit. The rms spot radius is measured from the centroid of the calculated ray positions, and is the size of the focused beam, ignoring diffraction. Note that the rms spot size is zero only in the paraxial approximation. The axial location of best focus was the location of the minimum rms spot radius. This plane was also considered the image plane for paraxial work. For the optimized system, the rms and Airy radii were 0.87 and $12.48 \mu \mathrm{m}$, respectively. The F-number of the optimized system was 19.37 .

It was not immediately evident whether the rms or Airy diameter, or some combination of the two, should drive the optimization of the optical system. Smaller rms spot radii are usually obtained with smaller beam diameters at the focusing lens. In contrast, for a fixed focal length, decreasing the beam diameter at the focusing lens results in a larger F-number, and, through Eq. (2), a larger Airy radius. The decision to design the optimized system around a minimized rms spot diameter was verified by examining the performance of various optical systems in a static test cell. That this was the correct choice was not evident a priori because of the complexity of the photoionization process and the abundance of small-scale structures in the Nd:YAG beam profile. To determine the correct merit function for the optimization, the threshold energy as a function of density in a static test cell was determined for several optical systems with varying beam diameters and F-numbers. The optimized system was also evaluated in the test cell for comparison.

The optical systems evaluated in the test cell were designed using paraxial techniques with the initial 4-mm-diam beam expanded via two lenses acting as a beam expander and then focused into the test cell with a third lens. Comparing systems with the same F-number but different beam diameters at the focusing lens separated the effects of the two parameters. A summary of the various systems is presented in Table 1. Different systems with the same F-number are designated short and long, referring to the relative focal length of the systems. The actual system F-numbers, listed in the table, varied slightly as lens spacing varied to accommodate mounting the lenses and alignment of the system. The tabulated value for the optimized system rms radius is slightly smaller than the value reported earlier because the effects of the beam passage through the facility window are not considered in the analysis of the systems evaluated in the test cell.

The static test cell consisted of a welded steel cube measuring $20 \mathrm{~cm}$ on a side. Plate glass windows on three sides of the box allowed optical access, with vacuum and air supply hoses attached to the fourth side. A pressure tap in the top of the test cell allowed measurement of the internal pressure. The test cell density was calculated from the test cell pressure and the room temperature, which was assumed to be constant at $297 \mathrm{~K}$. Although the densityin the test cell was regulated to be comparable to the freestream density in the Ludwieg tube, the test cell pressure was significantly higher than the facility freestream pressure, due to the static temperature difference between the quiescent test cell and the Mach- 4 nozzle flow. The test cell was located so that the laser beam passed through two of the windows on either side of the cell, with the perturbation-generating optics outside of one side of the cell and a beam dump terminating the beam on the other side. The formation of the disturbance was observed visually through the third window in the test cell, normal to the beam axis.

The threshold laser energy for formation of the perturbation is plotted vs the test cell density in Fig. 3 for the various optical 
Table 1 Test cell evaluation of various optical systems

\begin{tabular}{lcccccc}
\hline \hline System & $\begin{array}{c}\text { System } \\
\text { relative } \\
\text { length }\end{array}$ & $\begin{array}{c}\text { System } \\
\text { actual } \\
\text { F/\# }\end{array}$ & $\begin{array}{c}\text { System lens } \\
\text { focal lengths, } \\
\text { mm } \\
\text { (neg./pos./pos.) }\end{array}$ & $\begin{array}{c}\text { Beam } \\
\text { diameter } \\
\text { at focusing } \\
\text { lens, mm }\end{array}$ & $\begin{array}{c}\text { Airy } \\
\text { radius, } \\
\mu \mathrm{m}\end{array}$ & $\begin{array}{c}\text { RMS spot } \\
\text { radius, } \\
\mu \mathrm{m}\end{array}$ \\
\hline Optimized & & 19.37 & $-38 / 110 / 110$ & 10 & 12.48 & 0.694 \\
F/18.75 & & 17.77 & $-50 / 100 / 150$ & 8 & 11.51 & 0.895 \\
F/12.5 & Long & 12.0 & $-50 / 150 / 150$ & 12 & 7.76 & 2.008 \\
F/12.5 & Short & 11.82 & $-50 / 100 / 100$ & 8 & 7.66 & 0.922 \\
F/9.375 & & 8.88 & $-25 / 100 / 150$ & 16 & 5.69 & 10.81 \\
F/6.25 & Long & 6.04 & $-25 / 150 / 150$ & 24 & 3.84 & 29.41 \\
F/6.25 & Short & 6.07 & $-25 / 100 / 100$ & 16 & 3.88 & 15.32 \\
\hline \hline
\end{tabular}

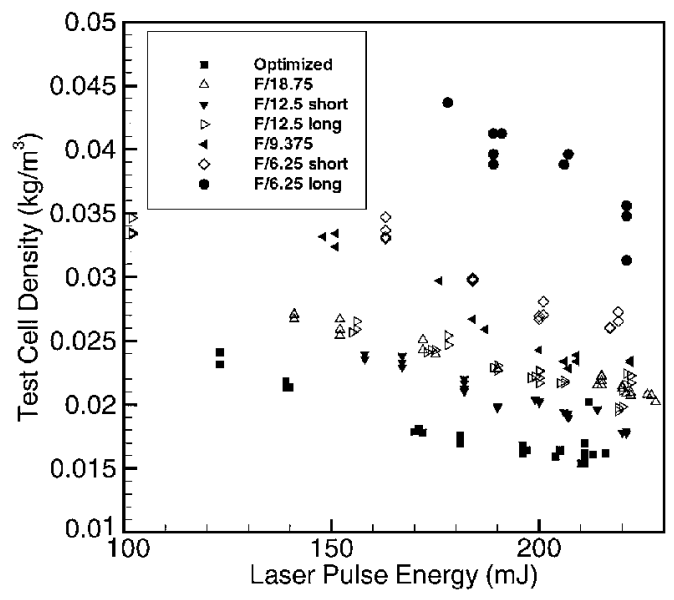

Fig. 3 Threshold density for ionization as a function of laser energy.

systems. For comparison with the data in Fig. 3, the freestream density in the Ludwieg tube nozzle is $0.032 \mathrm{~kg} / \mathrm{m}^{3}$ for driver-tube stagnation conditions of $97.9 \mathrm{kPa}$ and $297 \mathrm{~K}$. Estimation of the uncertainty in the ionization threshold measurements is difficult because the ionization was detected visually, a common detection method. ${ }^{7-9,11}$ The estimated maximum random error in the threshold density at a fixed laser pulse energy, determined by the largest spread between data points collected at similar conditions, is less than $25 \%$ of the threshold density. For most of the data, the spread between points collected under similar conditions is much less than the estimated maximum error.

The calculated spot rms and Airy radii are tabulated for each system in Table 1. Comparing the tabulated spot radii with the plotted density values at the ionization threshold, note that, for a fixed laser pulse energy, the minimum density for ionization decreases as the rms spot radius decreases and the F-number increases. Although the data for the F/ 18 system exhibits slightly higher threshold densities than the data for the short F/ 12 system, in apparent contradiction to the observed trend, note that the rms spot radii for the F/ 18 and short F/ 12 systems are comparable in size, and the differences between the two data sets are within the estimated error in the measurements. Thus, it may be concluded that system performance improves with smaller rms spot diameter. This trend is the opposite of that expected if the Airy radius were the important length scale in the focal region.

Based on these observations, it is clear that the design of this type of optical perturbation system for supersonic wind-tunnel applications must concentrate on obtaining the minimum possible rms spot diameter. This is due to the inverse relation between spherical aberration (the only aberration possible for the on-axis image point in the symmetric imaging system) and the cube of the F-number of the optical system. Because Airy radius is proportional to the F-number, the inverse cube behavior of spherical aberration drives the design. Hence, the minimum rms spot diameter should result in the maximum systemF-number. Additionally, the small length-scale structure inherentin the profile of a beam from an unstable-resonator Nd:YAG laser will diffract into a region substantially larger in radius than the Airy radius, reducing the importance of the theoretical Airy radius. It is likely that designing to minimize the rms spot radius leads to more sharply focused small-scale "hot-spots" in the

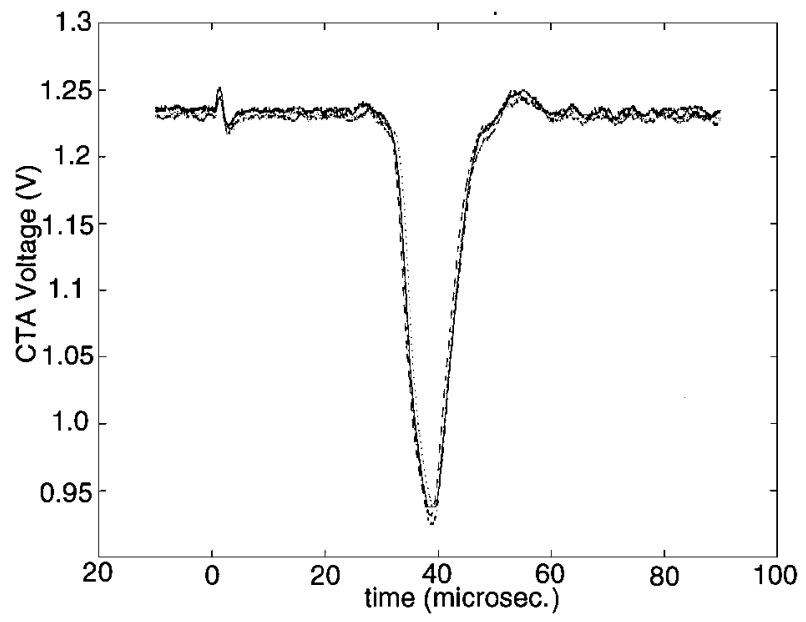

Fig. 4 First segment during supersonic flow for four runs over two days.

focus of the beam. Therefore, when exact ray trace methods are not employed, the largest system F-number that can be reasonably accommodated by the available space in the facility should be used. Similar results demonstrating the decrease in threshold intensity with increasing system F-number have been reported by Tambay and Thareja. ${ }^{11}$

\section{Characterization of the Disturbance}

Thermal spot perturbations were introduced into the Ludwieg tube nozzle flowfield using the optimized optical system described earlier. The symmetric bridge of a TSI IFA-100 constanttemperature anemometer (CTA) was used to characterize the passage of the thermal spot. Cold-wire constant-current anemometer measurements were attempted, but only to verify that the duration of the thermal spot passage was too brief for the bandwidth of the circuit. The laser power was measured prior to each run and assumed constant over the duration of the run. The CTA bridge voltage was digitally sampled using an eight-bit LeCroy 9304AM oscilloscope. Data were collected in segments of 5000 points at a sampling frequency of $50 \mathrm{MHz}$. Fifty segments were collected for each tube firing. The Q-switch signal from the laser was used to trigger each segment, so each segment corresponded to a single laser pulse. Typically, around 40 data segments were obtained during the supersonic portion of the run. Details of the experimentmay be found in Ref. 14.

The first data segment recorded during supersonic flow is plotted in Fig. 4 for four different tube firings. The noise associated with the laser firing, seen at time $t=0$, is convenient for visualizing the instant the laser fires. The reduction in CTA bridge voltage during passage of the thermal spot results from the decreased heat transfer from the wire during the passage of the less dense locally heated air. Because of the fixed-volume facility driver tube (stagnation chamber), the flow stagnation conditions are continually decreasing during the run. ${ }^{15}$ The perturbation strength, and thus the CTA response amplitude, attenuates with each successive disturbance during the run as the amount of energy transferred to the air decreases with the decrease in freestream density. Additionally, the time between the laser firing and the peak CTA response increases proportionally 
with the decrease in the square root of the stagnation temperature, indicating that the thermal spot convects with the local flow velocity. A detailed description of these observations is found in Ref. 16.

For application as a controlled perturbation, the laser-generated disturbance must be repeatable. As long as the room temperature, laser power, and stagnation conditions remain relatively constant, the perturbation is repeatable over several days without any adjustments made to the laser or the alignment of the optical system. The responses plotted in Fig. 4 are the first measured responses during supersonic flow for four runs collected over a two-day period. A total of 23 runs were made over both days. All traces were measured at the same location, although it should be noted that two of the runs were part of a transverse spot survey, and the optical system was translated along the beam axis between the various runs of the survey. From inspection, the responses seem to be very repeatable. Some slight scattermay be observed in the undisturbedvoltage level. This may be due to slight changes in the electrical properties of the filament as the wire ages. Statistical analysis of the CTA response to the thermal spot passage in a group of 10 sequential runs collected in a single day is reported in Ref. 12. Quantities such as the magnitude of the peak response and the time between the laser firing and the peak response were examined for both the first response during the run and the average of five sequential responses at various times during the run. In all cases, the standard deviation of the response magnitude was less than $1 \%$ of the mean value, whereas the standard deviation of the time between the laser firing and the peak response was less than $2 \%$ of the mean value.

The perturbation origin and the hot-wire probe could be moved with respect to each other by manipulating the traverses. The probe was moved vertically, whereas the perturbation was moved transversely, across the nozzle, by moving the small traverse supporting the optical system. The uncertainty in the measured traverse positions was less than $0.05 \mathrm{~mm}$ (Ref. 12). Surveys in the vertical and transverse directions were made to determine the approximate size of the perturbation. To ensure that any trends observed were the result of the perturbation and not any progressive offset within the system, the order in which the runs were collected was varied in a random fashion. The laser power and facility stagnation conditions were maintained constant within $2 \%$ of the mean value from run to run. Contours of constant deviation from the undisturbed mean are plotted for both a vertical and a transverse survey in Figs. 5 and 6 for initial driver tube pressures $P d$ of approximately $65.5 \mathrm{kPa}$ and ambient driver tube temperature and laser energy of approximately $200 \mathrm{~mJ} / \mathrm{pulse}$. The disturbance origin was $20 \mathrm{~mm}$ upstream of the probe. In both the transverse and vertical directions, the disturbance seems to be approximately $3 \mathrm{~mm}$ in diameter. At higher freestream densities, more gas is ionized, and the resulting thermal spot is larger. The thermal spot diameter is approximately $5 \mathrm{~mm}$ when the initial driver tube pressure is increased to $98 \mathrm{kPa}$ (Ref. 12). From inspection of the contours in Figs. 5 and 6, it may be concluded that

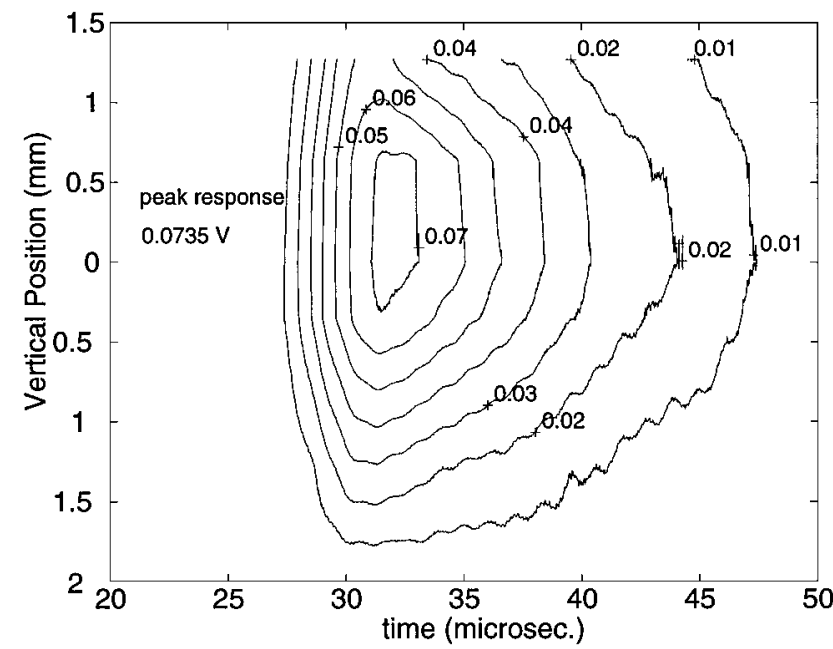

Fig. 5 Contours of constant response for the vertical survey $0.3 \mathrm{~s}$ after flow initiation; $P_{d} \approx 65.5 \mathrm{kPa}$.

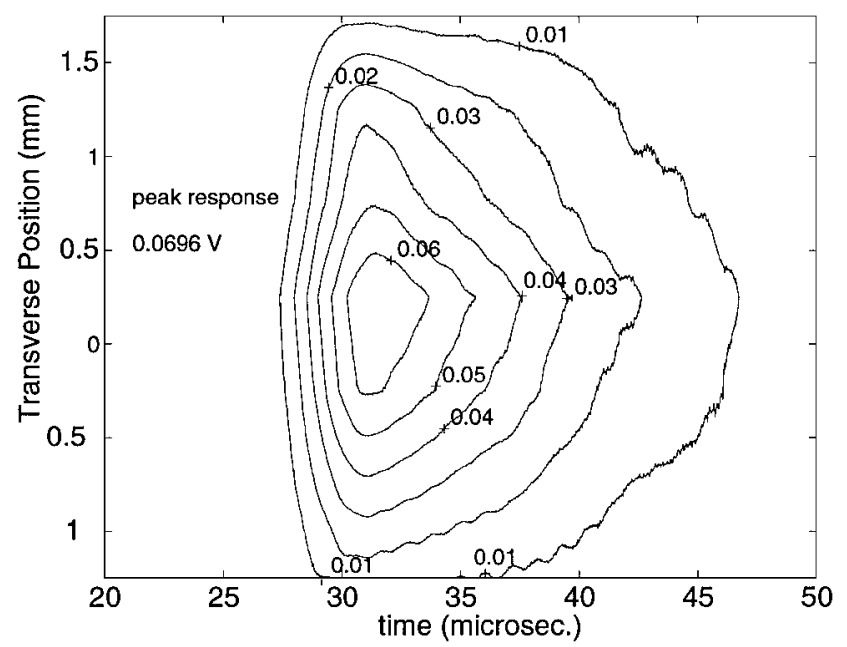

Fig. 6 Contours of constant response for the transverse survey $0.3 \mathrm{~s}$ after flow initiation; $P_{d} \approx 65.5 \mathrm{kPa}$.

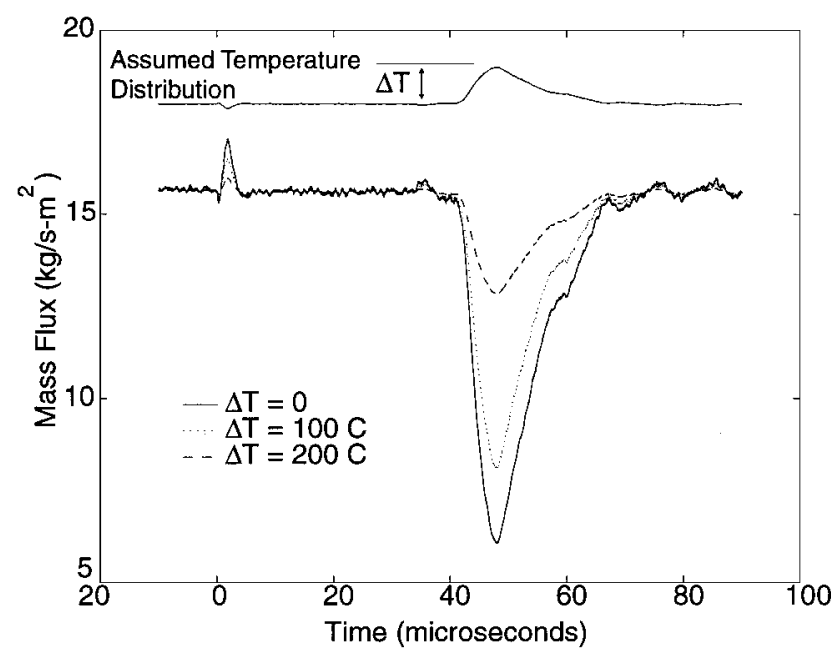

Fig. 7 Calculated mass flux near the thermal spot center $0.3 \mathrm{~s}$ after flow initiation.

the disturbance was axisymmetric with respect to the streamwise direction (the time axis). Because the timescales for the ionization process are several orders of magnitude less than the convective timescales of the flowfield, it may be argued that the formation of the disturbance occurred in the same fashion as in still air. If this is the case, the disturbance was also axisymmetric with respect to the beam axis, which in this application was approximately normal to the streamwise direction. Thus, it may be concluded that the disturbance at this point in the test section was approximately spherical. The spherical shape of the disturbance is not observed in the streamwise direction in the contours plotted in Figs. 5 and 6 due to the inadequate frequency response of the CTA. ${ }^{16}$

Knowledge of the local stagnation temperature is required for conversion of the CTA voltage to mass flux because the CTA is sensitive to both mass flux and stagnation temperature. ${ }^{17,18} \mathrm{Un}$ fortunately, the temperature increase induced by the thermal spot passage is unknown because the constant-current anemometer that was used to measure the local stagnation temperature lacked sufficient frequency response. ${ }^{12,14}$ Using an assumed spot temperature profile, limits on the magnitude of the mass-flux fluctuation were determined by converting to mass flux a CTA voltage trace corresponding to passage of the center of the thermal spot over the probe. The assumed temperature distribution had the same shape as the inverted CTA response to the thermal spot passage. The assumed temperature distribution with arbitrary amplitude is plotted above the calculated mass-flux profiles in Fig. 7, with the peak temperature induced by the disturbance denoted as $\Delta T$. Because both mass-flux and stagnation temperature fluctuations affect the CTA response, 
for the fixed response amplitude, the mass-flux deficit introduced by the thermal spot varies inversely with the temperature rise (Fig. 7). The solid line, labeled $\Delta T=0$, is the mass flux of the disturbance assuming the thermal spot causes no change from the freestream stagnation temperature. This line represents the upper bound of the mass-flux fluctuation. The peak mass-flux deficit resulting from the thermal spot in the limiting case is around $60 \%$ of the mean undisturbed mass flux. As the thermal spot peak temperature is increased to $\Delta T=200^{\circ} \mathrm{C}$, the peak mass-flux deficit decreases to around $20 \%$ of the undisturbed value. Thus, whereas accurate temperature data are required before the exact mass-flux fluctuation induced by the thermal spot may be calculated, it is evident that the thermal spot perturbations have substantial amplitude.

\section{Applications of the Laser Perturber}

To date, the laser perturber has been applied exclusively in the Mach-4 Purdue Quiet-Flow Ludwieg Tube, the facility for which it was developed. As noted earlier, the laser perturber was initially developed as a source of controlled freestream perturbations for boundary-layerreceptivity measurements. During development, the disturbancealso was applied to the flowfields generated by forwardfacing cavities and blunt bodies. Additional potential uses of the laser perturber include application as a flow-tagging mechanism for velocity measurements, use as a repeatable plasma source for recombination studies, and use as a freestream disturbance to examine the processing of disturbances by shock waves. In the applications described here, the laser perturber has been operated in flows with atmospheric stagnation conditions, although the system will also operate at subatmospheric stagnation pressures (see Figs. 5 and 6)

To examine the boundary-layer response to freestream disturbances, the laser-perturber was applied to the flowfield $7 \mathrm{~mm}$ upstream from the sharp tip of a 127-mm-long, 4:1 elliptic crosssection cone. ${ }^{14}$ The center of the thermal spot perturbation impacted the cone tip. The response of a hot wire (CTA) above the minor axis $125 \mathrm{~mm}$ from the cone tip to passage of the thermal spot in the cone boundary layer is shown in Fig. 8. The traces plotted in Fig. 8 are representative of the CTA responses in the regions near the wall, near the center and edge of the boundary layer, and above the boundary-layeredge. Note that, from the mean flow results reported in Refs. 12 and 14, the boundary-layeredge was determined to be between 4.6 and $5.1 \mathrm{~mm}$ from the cone surface, depending on the time during the run. The distance from the cone surface to the hot-wire probe is labeled near each trace. An important observation regarding the receptivity of the elliptic-cone boundary layer is noticeable when the CTA responses are compared to those measured in the freestream. In the freestream, the CTA response to the disturbance lasted on the order of tens of microseconds (see Fig. 7). In contrast, the duration of the CTA response to the thermal spot passage in the cone boundary layer is an order of magnitude larger, on the order of several hundred microseconds. Other information determined from measurement of the thermal spot in the boundary

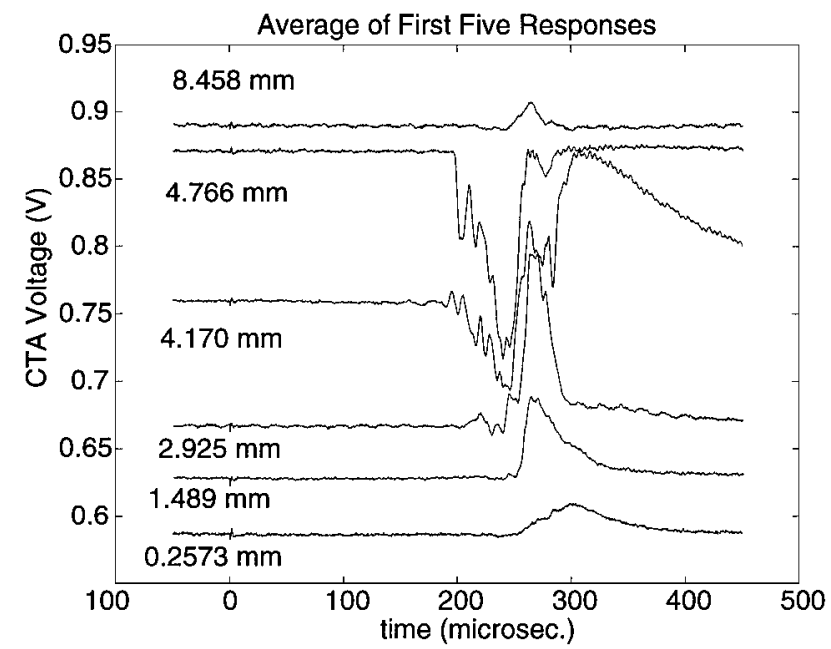

Fig. 8 Six representative CTA responses to the thermal spot at various distances from the cone surface. layer include the convection velocity and amplitude of disturbances as a function of location in the boundary layer. ${ }^{19}$

By introducing the repeatable thermal spot perturbationupstream of a forward-facingcavity in a blunt body, Ladoon et al. ${ }^{2}$ were able to measure and model the pressure fluctuations at the base of the cavity as a function of the cavity length-to-diameterratio $L / D$. The disturbance was introduced $22 \mathrm{~mm}$ upstream of the 19-mm-diam cylinder with a 9.5-mm-radiusnose tip and 9.5-mm-diam cavity. A plot of the typical fluctuating cavity base pressure induced by the introduction of the thermal spot is shown in Fig. 9 for two different values of $L / D$. The plot for $L / D=0$ reveals the fluctuating pressure in the stagnation region of a blunt nose. The oscillating pressure history shown in the plot for $L / D=1.064$ indicates the ringing of the cavity when excited by the thermal spot disturbance. The unique combination of the single controlled thermal spot perturbation and the low-noise quiet flow environment of the Purdue Quiet-Flow Ludwieg Tube ${ }^{2,15}$ allowed unambiguous characterization of the pressure fluctuations in the forward-facing cavity.

Similar use of the thermal spot perturbation has been made in the investigation of the subsonic region in the nose of a supersonic blunt body. ${ }^{1,20}$ The disturbance was introduced $21 \mathrm{~mm}$ upstream of the 25.4-mm-diam hemispheric nose. The pressure history near the stagnation point, obtained after introduction of the thermal spot upstream of the body, is plotted in Fig. 10. Note the similarity between
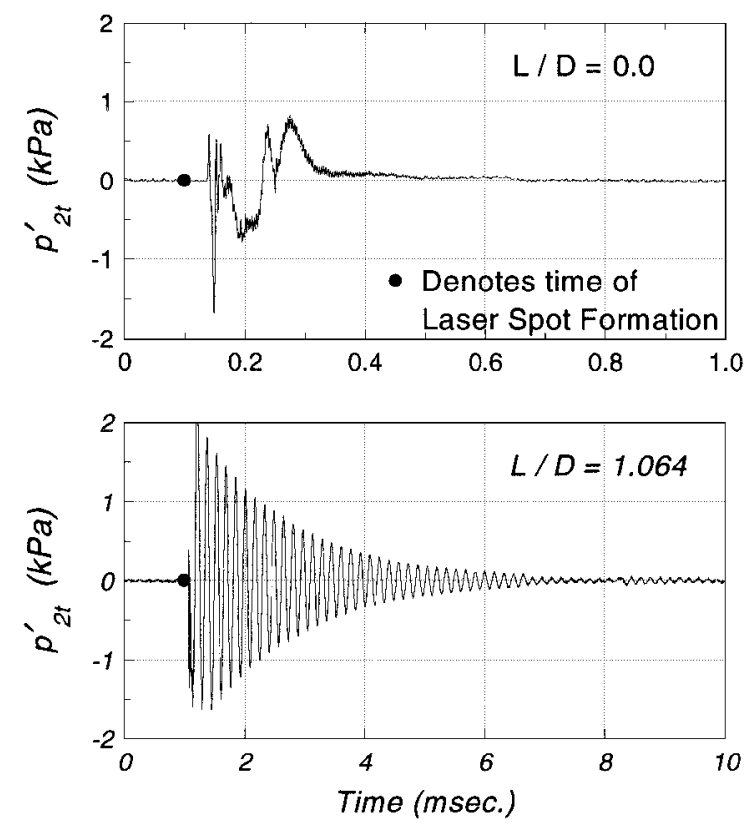

Fig. 9 Ringing of cavity in response to thermal spot (from Ref. 2).

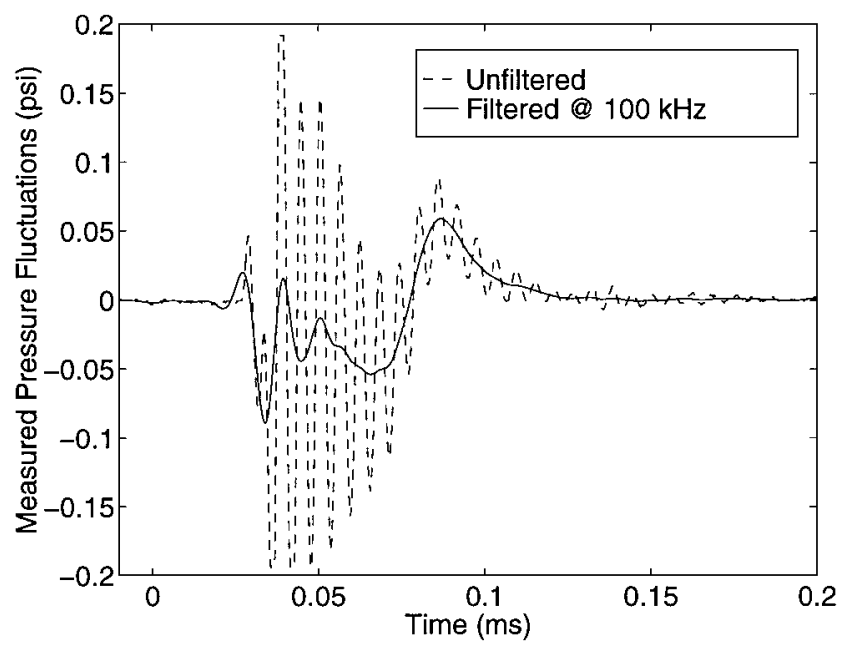

Fig. 10 Pressure fluctuation in response to laser perturbation (from Ref. 1). 
the filtered pressure signal measured on the blunt body in Fig. 10 and the pressure signal for the $L / D=0$ cavity configuration shown in Fig. 9. These two configurations are essentially the same flowfield, stagnation on a blunt body, with a few minor differences occurring in the diameters and nose radii of the two models.

\section{Conclusion}

A laser-generated, controlled, localized, freestream perturbation technique has been developed for use in supersonic and hypersonic flowfields. The thermal spot disturbance is formed after the plasma generated by a short-durationlaser pulse recombines into a region of locally heated air. Although CTA measurements of the convecting thermal spot are frequency-responselimited, the spatial variation of the measurements indicates that the thermal spot is approximately spherical. The diameter is several millimeters, with the exact value depending on the flow density. The thermal spot convects with the local freestream velocity.

A three-componentsystem of lenses is used to generate the thermal spot disturbance, although other lens combinations are possible. Investigation of the ionization of air as a function of density and energy intensity has revealed that the ionization threshold decreases with the geometric spot diameter at the focal point of the laser beam. Designing for maximum F-number appears to be a good rule of thumb for achieving minimum threshold intensities for a given gas density.

The laser perturber has been applied to investigations of boundary-layer receptivity and forward-facing cavity resonance in Mach4 flowfields. Another use of the laser perturber is as a flow tag, where the local velocity of the flowfield may be determined from the convection of the thermal spot.

\section{Acknowledgments}

This work was funded by the Air Force Office of Scientific Research under Contracts F49620-94-1-0067 and F49620-97-1-0037, monitored by Len Sakell. Additional funding for the facility was provided by gifts from The Boeing Company and gifts in memory of K. H. Hobbie. While at Purdue, tuition and salary for the first author were provided by the U.S. Air Force Palace Knight program. This research would not have been accomplished without the equipment built by the Purdue University Aerospace Sciences Laboratory Machine Shop, primarily Don Bower, Joe Zachary, Madeline Chadwell, and Jim Younts.

\section{References}

${ }^{1}$ Randall, L. A., "Receptivity Experiments on a Hemispherical Nose at Mach 4," M.S. Thesis, School of Aeronautics and Astronautics, Purdue Univ., W. Lafayette, IN, 1996.

${ }^{2}$ Ladoon, D., Schmisseur, J., and Schneider, S., "Physics of Resonance in a Supersonic Forward-Facing Cavity," Journal of Spacecraft and Rockets, Vol. 35, No. 5, 1998, pp. 626-632.
${ }^{3}$ Zel'dovich, Y. B., and Raizer, Y. P., Physics of Shock Waves and HighTemperature Hydrodynamic Phenomena, Academic, New York, 1996, pp. 338-343.

${ }^{4}$ Raizer, Y. P., Laser-Induced Discharge Phenomena, Consultants Bureau, New York, 1997, Chap. 1.

${ }^{5}$ Grey Morgan, C., "Laser-Induced Breakdown of Gases," Reports on Progress in Physics, Vol. 38, 1975, pp. 621-665.

${ }^{6}$ McGuire, J., "Fluid Dynamic Perturbations Using Laser Induced Breakdown," M.S. Thesis, School of Aeronautics and Astronautics, Purdue Univ., W. Lafayette, IN, Aug. 1994.

${ }^{7}$ Dewhurst, R., "Comparative Data on Molecular Gas Breakdown Thresholds in High Laser-Radiation Fields," Journal of Physics D, Vol. 11, No. 16 , 1978, pp. L191-195.

${ }^{8}$ Armstrong, R., Lucht, R., and Rawlins, W., "Spectroscopic Investigation of Laser-Initiated Low-Pressure Plasmas in Atmospheric Gases," Applied Optics, Vol. 22, No. 10, 1983, pp. 1573-1577.

${ }^{9}$ Sircar, A., Dwivedi, R., and Thareja, R., "Laser-Induced Breakdown of Ar, $\mathrm{N}_{2}$ and $\mathrm{O}_{2}$ Gases Using 1.064, 0.532, 0.355 and $0.266 \mu$ m Radiation," Applied Physics B: Lasers and Optics, Vol. 63, No. 6, 1996, pp. 623-627.

${ }^{10}$ Dewhurst, R., "Breakdown in the Rare Gases Using Single Picosecond Ruby Laser Pulses," Journal of Physics D, Vol. 10, No. 3, 1977, pp. 283-289.

${ }^{11}$ Tambay, R., and Thareja, R., "Laser-Induced Breakdown Studies of Laboratory Air at $0.266,0.355,0.532$, and $1.06 \mu \mathrm{m}$," Journal of Applied Physics, Vol. 70, No. 5, 1991, pp. 2890-2892.

${ }^{12}$ Schmisseur, J. D., "Receptivity of the Boundary Layer on a Mach4 Elliptic Cone to Laser-Generated Localized Freestream Perturbations," Ph.D. Dissertation, School of Aeronautics and Astronautics, Purdue Univ. W. Lafayette, IN, Dec. 1997.

${ }^{13}$ Zemax Optical Design Program User's Guide, Ver. 5.0, Focus Software, Inc., Tucson, AZ, 1995.

${ }^{14}$ Schmisseur, J., Schneider, S. P., and Collicott, S. H., "Receptivity of the Mach-4 Boundary Layer on an Elliptic Cone to Laser-Generated Localized Freestream Perturbations," AIAA Paper 98-0532, Jan. 1998

${ }^{15}$ Schneider, S., and Haven, C., "Quiet-Flow Ludwieg Tube for HighSpeed Transition Research," AIAA Journal, Vol. 33, No. 4, 1995, pp. 688693.

${ }^{16}$ Schmisseur, J., Collicott, S. H., and Schneider, S. P., "Laser-Generated Localized Freestream Perturbations in Supersonic/Hypersonic Flows," AIAA Paper 98-2495, June 1998.

${ }^{17}$ Smits, A., Hayakawa, K., and Muck, K., "Constant Temperature HotWire Anemometer Practice in Supersonic Flows," Experiments in Fluids, Vol. 1, No. 2, 1983, pp. 83-92.

${ }^{18}$ Spina, E., and McGinley, C., "Constant-Temperature Anemometry in Hypersonic Flow: Critical Issues and Sample Results," Experiments in Fluids, Vol. 17, No. 6, 1994, pp. 365-374.

${ }^{19}$ Schmisseur, J., Schneider, S. P., and Collicott, S. H., "Response of the Mach-4 Boundary-Layer on and Elliptic Cone to Laser-Generated Freestream Perturbations," AIAA Paper 99-0410, Jan. 1999.

${ }^{20}$ Schneider, S. P., Collicott, S. H., Schmisseur, J., Ladoon, D., Randall, L. A., Munro, S. E., and Salyer, T., "Laminar-Turbulent Transition Research in the Purdue Mach-4 Quiet-Flow Ludwieg Tube," AIAA Paper 96-2191, June 1996.

R. P. Lucht Associate Editor 